\title{
Effect of Density Gradient on the Acousto-Electric Wave Instability in Ion-Implanted Semiconductor Plasmas
}

\author{
S. Ghosh* and Pragati Khare \\ School of Studies in Physics, Vikram University, Ujjain 456010, India
}

(Received August 17, 2005)

\begin{abstract}
Using hydrodynamic model of inhomogeneous plasma, an analytical investigation of excitation of acousto-electric mode was made in $n$-type piezoelectric ion-implanted semiconductor plasma. By employing the multi-fluid balance equations along with the elastic and Maxwell equations, a compact dispersion relation for the cases in which colloidal grains are either stationary $\left(\vartheta_{0 d}=0\right)$ or streaming $\left(\vartheta_{0 d} \neq 0\right)$ is derived. We find that the choice of homogeneous medium is favorable in achieving higher acoustic gain per radian and the results of the investigation should be useful in understanding the characteristics of longitudinal acousto-electric wave in ion-implanted piezoelectric semiconductor whose main constituents are electrons and negatively charged colloidal particles.
\end{abstract}

PACS numbers: 61.72.Vv, 72.30.+q, 82.70.Dd

\section{Introduction}

Physics of colloids laden semiconductor plasma is recently studied rather intensively because of its importance for a number of applications in processing of materials, fabrication of devices, etc. [1-3]. The influence of the highly charged and massive colloids, formed within the ion-implanted semiconductor material, on the plasma properties, wave propagation characteristics, in finding the novel wave modes and in modification of existing plasma mode spectra is a subject of great current interest. Recently, this medium has attracted plasma and device physicists in finding the new modes of electro-acoustic [4], Alfven [5], electro-kinetic waves [6] and instability of acousto-electric wave [7] in colloids laden or ion-implanted semiconductor plasmas.

In the last decade, most of the earlier investigations of phonon-plasmon interaction have been made for homogeneous semiconductors [8-12]. Under crossed

*corresponding author; e-mail: drsanjayghosh@rediffmail.com 
fields configurations complete homogeneity of a plasma system cannot be achieved due to the Lorentz force. Also it is practically impossible to develop a complete homogeneous crystal medium. On the other hand, the inhomogeneity may be induced in a material by non-uniform doping or by exposing it to non-uniform radiation. If the system has gradient of density, temperature, pressure, magnetic field etc. a plasma current or a particle drift exists otherwise in the presence of dc electric field the gradient enhances the particle drift.

The interaction between the electron streams and various waves present in an inhomogeneous system have aroused great interest because under certain conditions amplification or generation of various types of waves, both electromagnetic [13-15] and acoustic is easily possible in such system [16, 17]. Guha et al. have analyzed the theory of acoustic wave amplification in an inhomogeneous semiconductor $[17,18]$.

Very recently, the present authors have reported an analytical study on excitation of modified electro-acoustic modes and its absorption characteristics in $n$-type homogeneous semiconductor plasma embedded with colloidal grains [4]. In this report authors found a strong resonant interaction between the acoustic mode and a colloidal particles-modified electro-kinetic mode due to the piezoelectric nature of the medium. Working in the same direction, in the present paper we focused our attention on the acousto-electric wave interaction in inhomogeneous semiconductor plasmas.

Motivated by the fascinating works of Ghosh et al. $[4,17,18]$ in the present paper we intend to report the investigations made on the dispersion and absorption characteristics of phonon-plasmon mode in inhomogeneous colloids laden semiconductor plasma. The interaction between electrons and lattice vibrations is one of the fundamental interaction processes in solids; it gives useful information regarding the physical properties of the host medium. By the application of a dc electric field the acousto-electric interaction can lead to amplification of acoustic wave which has been utilized for the fabrication of delay lines, acousto-electric amplifiers and oscillators etc. As far as our knowledge goes, no study has yet been reported on the acousto-electric wave interaction in colloids laden inhomogeneous piezoelectric semiconductor plasmas.

The manuscript is summarized as follows. In Sect. 2, we present the theoretical model and derive a dispersion relation for acousto-electric wave interaction in the ion implanted semiconductor plasmas. In Sect. 3, we present numerical appreciation and discussions. In Sect. 4 we briefly conclude the results obtained.

\section{Theoretical model}

In the present model, we considered an inhomogeneous piezoelectric semiconductor sample of infinite extent in the presence of implanted ions which consequently turn to neutral colloidal particles. The medium is subjected to a dc electric field applied along the $z$-axis. In the present paper, we have analyzed the role of 
inhomogeneity due to the density gradient on the acoustic wave propagation which is taken to be propagating along the $z$-axis and the density gradient $\nabla n_{0}$ is taken in the direction of wave propagation. Since the electrons move swiftly in presence of dc electric field therefore we considered that the neutral colloids tend to acquire a negative charge due to various sticking processes. The average size of the colloids is assumed to be much less than the inter-grain distance, the electron Debye radius, as well as the wavelength, so that they can be treated as point masses [19]. Hence, the material can be safely treated as the multi-component plasma consisting of electrons and negatively charged colloids under hydrodynamic limit.

For the wave, we assume that all perturbations vary as $\exp [\mathrm{i}(\omega t-\kappa z)]$ in which $(\omega, \kappa)$ are the frequency and wave number. Under the considered geometry, the wave equation in an elastic piezoelectric medium becomes

$$
\left(-\rho \omega^{2}+c \kappa^{2}\right) u_{x}=\mathrm{i} \kappa \beta E_{z},
$$

where $\beta$ is the piezoelectric coefficient of the medium and all other symbols have the same meanings as defined by Steele and Vural [20].

In deriving Eq. (1) we have assumed that the solid is piezoelectric and has cubic symmetry. This assumption simplifies the tensor components of relevant physical parameters without diluting the physical significance of the interactions to be studied. The acoustic wave is taken as a shear wave that propagates in the $z$ direction, which in this case is a $\langle 011\rangle$ axis of the crystal. The lattice displacement $\boldsymbol{u}$ is taken only in the $x$ direction, which is a $\langle 100\rangle$ axis. For such a wave there will be an electric field component in the $\boldsymbol{k}$ direction. This geometry is appropriate to many piezoelectric semiconductors of group III-V.

If the wavelength is much smaller than the density decay length $\delta^{-1}(\delta=$ $\Delta n_{0} / n_{0}$ ), we can make the "local approximation" by assuming $\boldsymbol{k}$ independent of $z$. Then, for electrons the continuity equation yields

$$
\frac{n_{1}}{n_{0}}=\frac{(\kappa+\mathrm{i} \delta) \vartheta_{1 e}}{\omega-\vartheta_{0 e}(\kappa-\mathrm{i} \delta)} .
$$

Since the colloidal particles are independent of the above approximation, therefore, for negatively charged colloids the continuity equation yields

$$
\frac{n_{1}}{n_{0}}=\frac{\kappa \vartheta_{1 d}}{\left(\omega-\kappa \vartheta_{0 d}\right)} .
$$

In the present configuration the electrons and charged colloids acquire perturbed motion in accordance with first-order momentum balance equation and we can write

$$
\vartheta_{e z}=\frac{\mathrm{i}\left(e / m_{e}\right) E_{1 z}}{F(\omega, \kappa)}
$$

and

$$
\vartheta_{d z}=\frac{-\mathrm{i}\left(z_{d} e / m_{d}\right) E_{1 z}}{G(\omega, \kappa)},
$$

where 


$$
F(\omega, \kappa)=\omega-\kappa \vartheta_{0 e}-\mathrm{i} \nu_{e}-\frac{D \nu_{e} \kappa(\kappa+\mathrm{i} \delta)}{\omega-\vartheta_{0 e}(\kappa-\mathrm{i} \delta)},
$$

and

$$
G(\omega, \kappa)=\omega-\kappa \vartheta_{0 d}-\mathrm{i} \nu_{d}
$$

Here $e$ and $z_{d} e$ are the charges on electrons and colloids, respectively. $z_{d}=q_{d} / e$ is the ratio of the charges, $q_{d}$ on a colloidal grain to the electron charge $e . \vartheta_{0 d}$ and $\nu_{d}$ are the drift velocity and momentum transfer collision frequency of the colloids. All other symbols have their usual meanings as defined by Steele and Vural [20].

Using Eqs. (1) to (7), we get the dispersion relation for phonon-plasmon interactions in inhomogeneous piezoelectric semiconductor plasma as

$$
\begin{aligned}
& \left(\omega^{2}-\kappa^{2} \vartheta_{s}^{2}\right)\left\{1-\frac{\omega_{p e}^{2}(\kappa+\mathrm{i} \delta)}{\kappa\left[\omega-\vartheta_{0 e}(\kappa-\mathrm{i} \delta)\right] F(\omega, \kappa)}-\frac{\omega_{p d}^{2}}{\left(\omega-\kappa \vartheta_{0 d}\right) G(\omega, \kappa)}\right\} \\
& =K^{2} \kappa^{2} \vartheta_{s}^{2},
\end{aligned}
$$

where $K^{2}=\beta^{2} / c \varepsilon$ is the dimensionless electromechanical coupling coefficient. The plasma frequencies for electrons and colloids are given as $\omega_{p e}=\sqrt{\frac{e^{2} n_{0 e}}{\varepsilon m_{e}}}$ and $\omega_{p d}=\sqrt{\frac{z_{d}^{2} e^{2} n_{0 d}}{\varepsilon m_{d}}}$ in which $n_{0 e, d}$ are the unperturbed number densities of electrons and colloids, respectively.

In absence of density gradient $(\delta=0)$ and charged colloids $\left(\omega_{p d}=0\right)$, Eq. (8) reduces to Eq. (8-20) of Steele and Vural [20] and represents the phonon-plasmon interaction in piezoelectric semiconductor plasma.

In the collision dominated regime $\left(\omega \ll \nu_{e}, \nu_{d}\right.$ and $\left.\kappa \vartheta_{0 e} \ll \nu_{e}, \kappa \vartheta_{0 d} \ll \nu_{d}\right)$ under the approximation $\kappa \vartheta_{s} / \omega=1+\mathrm{i} \alpha$ [21], where the gain per radian $\alpha$ is $\ll 1$, Eq. (8) yields

$$
\begin{aligned}
\alpha= & \frac{1}{2} K^{2} \gamma_{e} \frac{\omega_{R e}}{\omega}(A+B)^{-1}\left\{1+\frac{\omega_{R d} \gamma_{e}}{\omega_{R e} \gamma_{d}}+\frac{\omega_{R d}}{\omega_{R e}} \frac{\omega^{2}}{\omega_{D}^{2} \gamma_{e} \gamma_{d}}\right. \\
& -2 \frac{\delta}{\kappa} \frac{\omega_{R d}}{\omega_{R e}}\left(\frac{2 \omega}{\omega_{D} \gamma_{d}}+\frac{\omega}{\omega_{D} \gamma_{e} \gamma_{d}}\right) \\
& \left.-\left(\frac{\delta}{\kappa}\right)^{2}\left[\frac{\gamma_{e}+1}{\gamma_{e}}\left(1-\frac{\omega_{R d}}{\omega_{R e}} \frac{\gamma_{e}+1}{\gamma_{d}}\right)-\frac{\omega_{R d}}{\omega_{R e}} \frac{\omega^{2}}{\omega_{D}^{2} \gamma_{e} \gamma_{d}}\right]\right\}
\end{aligned}
$$

in which $\omega_{D}=\vartheta_{s}^{2} / D$ is the electron diffusion frequency, $\gamma_{e, d}=\vartheta_{0 e, d} / \vartheta_{s}-1$, $\omega_{R e, d}=\omega_{p e, d}^{2} / \nu_{e, d}$. The sound wave is amplified only when gain per radian $\alpha$ obtained in Eq. (9) is positive.

Now we shall focus our attention towards the amplification characteristics of sound wave for two distinct cases i.e. of stationary and streaming charged colloids.

\subsection{Case 1: stationary colloids $\left(\vartheta_{0 d}=0\right)$}

It is a well-known fact that unless one considers the lowest part of the grain mass spectrum and very low frequency modes, the conclusion is that the grain 
dynamics can be ignored with respect to the electron dynamics [22]. Thus for ultrasonic frequency regime and robust colloidal grains, we can safely assume $\vartheta_{0 d}=0$ and consequently $\gamma_{d}=-1$. Using this approximation Eq. (9) reduces to

$$
\alpha \approx \frac{\frac{1}{2} K^{2} \gamma_{e} \frac{\omega_{R e}}{\omega}\left\{1-\frac{\omega_{R d} \gamma_{e}}{\omega_{R e}}\left[1+\left(\frac{\omega}{\omega_{D} \gamma_{e}}\right)^{2}-2 \frac{\delta}{\kappa}\left(\frac{2 \omega}{\omega_{D} \gamma_{e}}+\frac{\omega}{\omega_{D} \gamma_{e}^{2}}\right)\right]\right\}}{A+B},
$$

where

$$
A=\left(\frac{\omega_{R e}}{\omega}\right)^{2}\left[1+\frac{\omega^{2}}{\omega_{D} \omega_{R e}}-\frac{\omega_{R d} \gamma_{e}}{\omega_{R e}}-\frac{\delta}{\kappa} \frac{\omega_{R d}}{\omega_{R e}} \omega\left(\frac{\gamma_{e}+1}{\omega_{R d}}-\frac{1}{\omega_{D}}\right)\right]^{2},
$$

and

$$
B=\gamma_{e}^{2}\left\{1+\frac{\omega_{R d}}{\omega_{D} \gamma_{e}}-\frac{\delta}{\kappa} \frac{1}{\gamma_{e}}\left[\frac{\omega}{\omega_{D}}+\frac{\omega_{R e}}{\omega}+\frac{\omega_{R d}\left(\gamma_{e}+1\right)}{\omega}\right]\right\}^{2}
$$

From Eq. (10a) it may be inferred that this mode will be amplified only when

$$
\left|\gamma_{e}\right|>0
$$

and

$$
\omega<\omega_{D}\left|\gamma_{e}\right|\left[\sqrt{\frac{\omega_{R e}}{\omega_{R d}\left|\gamma_{e}\right|}-1}+\frac{\delta}{\kappa}\left(2+\frac{1}{\left|\gamma_{e}\right|}\right)\right] .
$$

It is clear from numerator of Eq. (10) that the inhomogeneity factor $(\delta / \kappa)$ tends to decrease the value of gain per radian. It may also be inferred from Eq. (10b) that the limiting value of frequency below which one gets amplification, increases further in presence of inhomogeneity $(\delta / \kappa)$; hence the wave frequency spectrum width for which one gets acoustic gain enhances. Thus it may be concluded that the presence of inhomogeneity in the medium effectively modifies the instability criteria.

\subsection{Case 2: streaming colloids $\left(\vartheta_{0 d} \neq 0\right)$}

In this case we consider the dynamics of colloidal grains. To study the amplification characteristics of the acoustic wave, we will discuss different velocity regimes as follows.

2.2.1. When $\gamma_{e}>0$ and $\gamma_{d}>0$ i.e. $\vartheta_{0 e}>\vartheta_{s}<\vartheta_{0 d}$

In this regime, the expression for gain per radian (Eq. (9)) reduces to

$$
\alpha=
$$

$$
\frac{\frac{1}{2} K^{2}\left|\gamma_{e}\right| \frac{\omega_{R e}}{\omega}\left\{1+\frac{\omega_{R d}}{\omega_{R e}}\left|\frac{\gamma_{e}}{\gamma_{d}}\right|\left[1+\left(\frac{\omega}{\omega_{D}\left|\gamma_{e}\right|}\right)^{2}-2 \frac{\delta}{\kappa}\left(\frac{2 \omega}{\omega_{D}\left|\gamma_{e}\right|}+\frac{\omega}{\omega_{D}\left|\gamma_{e}\right|^{2}}\right)\right]\right\}}{A+B} .
$$

It may be inferred from the above equation that acoustic wave will be of amplifying nature only when

$$
1+\left(\frac{\omega}{\omega_{D}\left|\gamma_{e}\right|}\right)^{2}>2 \frac{\delta}{\kappa}\left(\frac{2 \omega}{\omega_{D}\left|\gamma_{e}\right|}+\frac{\omega}{\omega_{D}\left|\gamma_{e}\right|^{2}}\right)
$$

Thus the gain per radian in this velocity region will be 


$$
\alpha \approx \frac{\frac{1}{2} K^{2}\left|\gamma_{e}\right| \frac{\omega_{R e}}{\omega}\left\{1+\frac{\omega_{R d}}{\omega_{R e}}\left|\frac{\gamma_{e}}{\gamma_{d}}\right|\left[1+\left(\frac{\omega}{\omega_{D}\left|\gamma_{e}\right|}\right)^{2}\right]\right\}}{A+B},
$$

where

$$
A=\left(\frac{\omega_{R e}}{\omega}\right)^{2}\left[1+\frac{\omega^{2}}{\omega_{D} \omega_{R e}}+\frac{\omega_{R d}}{\omega_{R e}}\left|\frac{\gamma_{e}}{\gamma_{d}}\right|-\frac{\delta}{\kappa} \frac{\omega_{R d}}{\omega_{R e}} \omega\left(\frac{\left|\gamma_{e}\right|+1}{\omega_{R d}}+\frac{1}{\omega_{D}\left|\gamma_{d}\right|}\right)\right]^{2}
$$

and

$$
B=\left|\gamma_{e}\right|^{2}\left\{1-\frac{\omega_{R d}}{\omega_{D}\left|\gamma_{e}\right|\left|\gamma_{d}\right|}-\frac{\delta}{\kappa} \frac{1}{\left|\gamma_{e}\right|}\left[\frac{\omega}{\omega_{D}}+\frac{\omega_{R e}}{\omega}-\frac{\omega_{R d}\left(\left|\gamma_{e}\right|+1\right)}{\omega\left|\gamma_{d}\right|}\right]\right\}^{2} .
$$

One can infer from Eq. (11b) that in this velocity regime the wave will always be of amplifying nature but the value of gain per radian is modified due to the presence of density gradient in this velocity regime.

2.2.2. When $\gamma_{e}>0$ and $\gamma_{d}<0$ i.e. $\vartheta_{0 e}>\vartheta_{s}>\vartheta_{0 d}$

From Eq. (9) one can find the gain per radian for this velocity regime as $\alpha=$

$$
\frac{\frac{1}{2} K^{2}\left|\gamma_{e}\right| \frac{\omega_{R e}}{\omega}\left\{1-\frac{\omega_{R d}}{\omega_{R e}}\left|\frac{\gamma_{e}}{\gamma_{d}}\right|\left[1+\left(\frac{\omega}{\omega_{D}\left|\gamma_{e}\right|}\right)^{2}-2 \frac{\delta}{\kappa}\left(\frac{2 \omega}{\omega_{D}\left|\gamma_{e}\right|}+\frac{\omega}{\omega_{D}\left|\gamma_{e}\right|^{2}}\right)\right]\right\}}{A+B} .
$$

From Eq. (12) one can infer that $\alpha$ will be positive only when the expression within curly bracket in numerator is positive. Thus the gain per radian in this velocity region will be

where

$$
\alpha \approx \frac{\frac{1}{2} K^{2}\left|\gamma_{e}\right| \frac{\omega_{R e}}{\omega}}{A+B}
$$

$$
A=\left(\frac{\omega_{R e}}{\omega}\right)^{2}\left[1+\frac{\omega^{2}}{\omega_{D} \omega_{R e}}-\frac{\omega_{R d}}{\omega_{R e}}\left|\frac{\gamma_{e}}{\gamma_{d}}\right|-\frac{\delta}{\kappa} \frac{\omega_{R d}}{\omega_{R e}} \omega\left(\frac{\left|\gamma_{e}\right|+1}{\omega_{R d}}-\frac{1}{\omega_{D}\left|\gamma_{d}\right|}\right)\right]^{2},
$$

and

$$
B=\left|\gamma_{e}\right|^{2}\left\{1+\frac{\omega_{R d}}{\omega_{D}\left|\gamma_{e}\right|\left|\gamma_{d}\right|}-\frac{\delta}{\kappa} \frac{1}{\left|\gamma_{e}\right|}\left[\frac{\omega}{\omega_{D}}+\frac{\omega_{R e}}{\omega}+\frac{\omega_{R d}\left(\left|\gamma_{e}\right|+1\right)}{\omega\left|\gamma_{d}\right|}\right]\right\}^{2} .
$$

In this case even though the charged colloids move slowly in comparison with acoustic phonon speed, one gets the amplification of sound wave in a frequency regime given as

$$
\omega<\omega_{D}\left|\gamma_{e}\right|\left[\sqrt{\frac{\omega_{R e}}{\omega_{R d}}\left|\frac{\gamma_{d}}{\gamma_{e}}\right|-1}+\frac{\delta}{\kappa}\left(2+\frac{1}{\left|\gamma_{e}\right|}\right)\right] .
$$

It may be inferred here that gain per radian in the inhomogeneous medium decreases and simultaneously frequency spectrum width for which one finds gain, increases due to presence of density gradient. Hence the amplification characteristics of acoustic wave in this velocity regime is always decided by the physical parameters of the non-uniform semiconductor medium. 
2.2.3. When $\gamma_{e}<0$ and $\gamma_{d}>0$ i.e. $\vartheta_{0 e}<\vartheta_{s}<\vartheta_{0 d}$

In this velocity regime, Eq. (9) yields that positive $\alpha$ is possible only when

$$
\omega>\omega_{D}\left|\gamma_{e}\right|\left[\sqrt{\frac{\omega_{R e}}{\omega_{R d}}\left|\frac{\gamma_{d}}{\gamma_{e}}\right|-1}-\frac{\delta}{\kappa}\left(2-\frac{1}{\left|\gamma_{e}\right|}\right)\right],
$$

and the value of gain per radian will be

$$
\alpha \approx \frac{\frac{1}{2} K^{2}\left|\gamma_{e}\right| \frac{\omega_{R e}}{\omega}\left\{\frac{\omega_{R d}}{\omega_{R e}}\left|\frac{\gamma_{e}}{\gamma_{d}}\right|\left[1+\left(\frac{\omega}{\omega_{D}\left|\gamma_{e}\right|}\right)^{2}+2 \frac{\delta}{\kappa}\left(\frac{2 \omega}{\omega_{D}\left|\gamma_{e}\right|}-\frac{\omega}{\omega_{D}\left|\gamma_{e}\right|^{2}}\right)\right]\right\}}{A+B},
$$

where

$$
A=\left(\frac{\omega_{R e}}{\omega}\right)^{2}\left[1+\frac{\omega^{2}}{\omega_{D} \omega_{R e}}-\frac{\omega_{R d}}{\omega_{R e}}\left|\frac{\gamma_{e}}{\gamma_{d}}\right|+\frac{\delta}{\kappa} \frac{\omega_{R d}}{\omega_{R e}} \omega\left(\frac{\left|\gamma_{e}\right|-1}{\omega_{R d}}-\frac{1}{\omega_{D}\left|\gamma_{d}\right|}\right)\right]^{2}
$$

and

$$
B=\left|\gamma_{e}\right|^{2}\left\{1+\frac{\omega_{R d}}{\omega_{D}\left|\gamma_{e}\right|\left|\gamma_{d}\right|}+\frac{\delta}{\kappa} \frac{1}{\left|\gamma_{e}\right|}\left[\frac{\omega}{\omega_{D}}+\frac{\omega_{R e}}{\omega}+\frac{\omega_{R d}\left(\left|\gamma_{e}\right|-1\right)}{\omega\left|\gamma_{d}\right|}\right]\right\}^{2} .
$$

Even though the electron drift is smaller than the acoustic speed, one may get the sound amplification in inhomogeneous semiconductor plasma where the inhomogeneity is due to the density gradient. Hence this is a new amplifying mode in phonon-plasmon interaction in piezoelectric semiconductor plasma.

\subsubsection{When $\gamma_{e}<0$ and $\gamma_{d}<0$ i.e. $\vartheta_{0 e}<\vartheta_{s}>\vartheta_{0 d}$}

In this velocity regime one will always get decayed mode $(\alpha<0)$; hence it is of no importance in this report.

\section{Results and discussions}

To have numerical appreciations of the result obtained in the previous section, we made calculations to study amplification characteristics of acoustic mode supported by the colloids contaminated plasma present in an inhomogeneous semiconductor viz. InSb. The parameters chosen are: $m_{e}=0.014 m_{0}, m_{0}$ being the electron free mass, $m_{d}=10^{-27} \mathrm{~kg}, \varepsilon_{L}=17.54, \beta=0.054 \mathrm{C} \mathrm{m}^{-2}$, $\rho=5.8 \times 10^{3} \mathrm{~kg} \mathrm{~m}{ }^{-3}, n_{0 e}=10^{24} \mathrm{~m}^{-3}, n_{0 d}=10^{18} \mathrm{~m}^{-3}, \nu_{e}=3.5 \times 10^{11} \mathrm{~s}^{-1}$ and $\nu_{d}=3.248 \times 10^{10} \mathrm{~s}^{-1}$. We have used Eqs. (10), (11a), (12) and (15) for the numerical calculations and the results are displayed in Figs. 1-4.

For stationary colloids (case 1), Fig. 1 illustrates the absorption characteristics of acoustic mode using density gradient $(\delta)$ as a parameter. It is clear from the graph that for all values of $\delta$ the gain per radian $(\alpha)$ follows the identical nature of variation with wave frequency $(\omega)$ i.e. the gain first increases with increase in frequency, achieves a maximum value and then starts decreasing with increasing frequency. For $\omega \geq 2.214 \times 10^{11} \mathrm{~s}^{-1}$ the characteristics of the mode is reversed and it starts showing decaying nature for all possible values of $\delta$. It means that to get amplification of acoustic mode in presence of stationary colloids, one has to confine in a frequency regime for which $\omega<2.214 \times 10^{11} \mathrm{~s}^{-1}$. Hence, one may define a critical frequency $\omega_{\mathrm{cr}} \approx 2.214 \times 10^{11} \mathrm{~s}^{-1}$, which is the reversal point of 


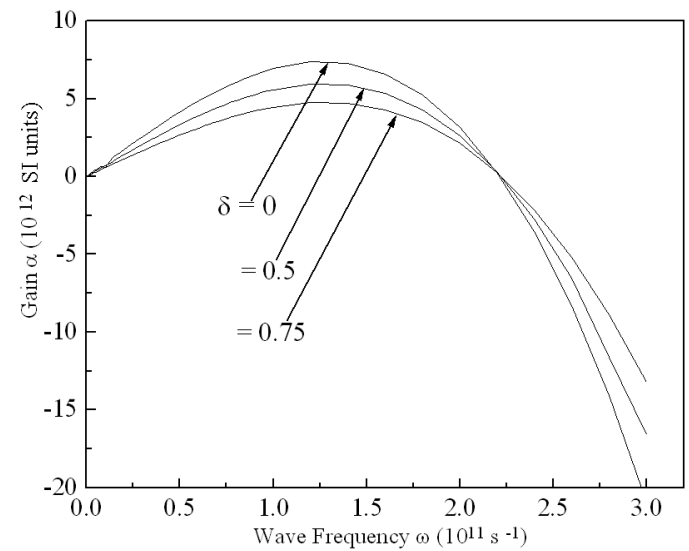

Fig. 1. Variation of gain $\alpha$ with wave frequency $\omega$ using $\delta$ as parameter at $E_{0}=$ $10^{3} \mathrm{~V} \mathrm{~m}^{-1}$ for stationary colloids (see Sect. 2.1).

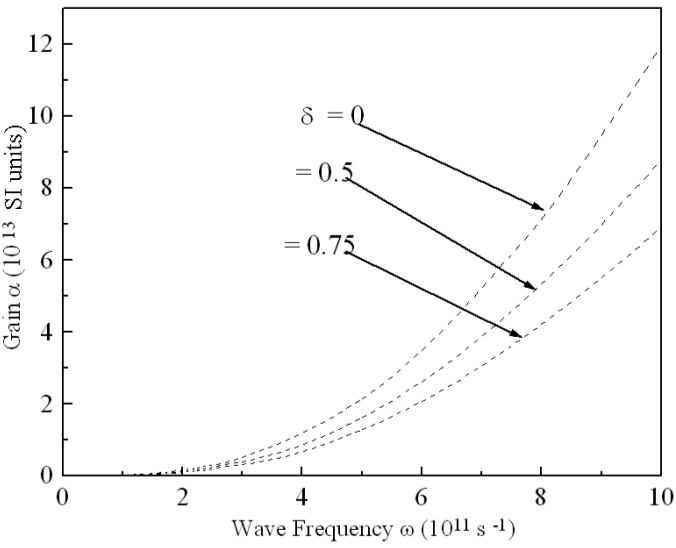

Fig. 2. Variation of gain $\alpha$ with wave frequency $\omega$ using $\delta$ as parameter at $E_{0}=$ $10^{3} \mathrm{~V} \mathrm{~m}^{-1}$ for $\vartheta_{0 e}>\vartheta_{s}<\vartheta_{0 d}$ (see Sect. 2.2.1).

the wave amplification nature. It may also be inferred from the graph that the acoustic gain per radian is always larger in homogeneous medium $(\delta=0)$ than in inhomogeneous media $(\delta \neq 0)$. Consequently, the maximum gain obtained in homogeneous sample $\left(\alpha_{\max } \approx 7.3 \times 10^{12}\right.$ SI units at $\left.\omega \approx 1.5 \times 10^{11} \mathrm{~s}^{-1}\right)$ is the largest. But the value of wave frequency at which one gets maximum gain $\left(\alpha_{\max }\right)$ is found to be shifted towards higher frequency as $\delta$ increases. Hence in non-uniform medium in the presence of stationary colloids the value of gain constant and the maximum achievable gain decrease. Thus, the homogeneous medium is always the best choice for obtaining the maximum value of acoustic gain per radian in colloids laden semiconductor plasmas.

Figure 2 infers that in the velocity regime $\vartheta_{0 e}>\vartheta_{s}<\vartheta_{0 d}$ the acoustic mode is always of amplifying nature for the frequency regime under study. With the 


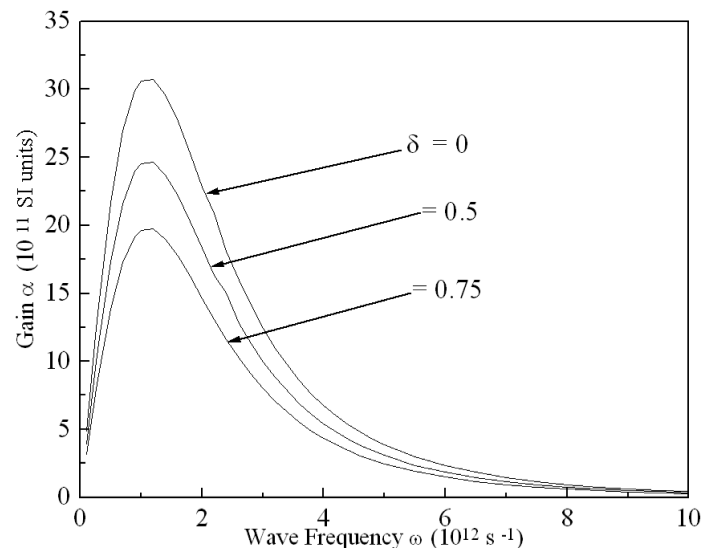

Fig. 3. Variation of gain $\alpha$ with wave frequency $\omega$ using $\delta$ as parameter at $E_{0}=$ $5 \times 10^{3} \mathrm{~V} \mathrm{~m}^{-1}$ for $\vartheta_{0 e}>\vartheta_{s}>\vartheta_{0 d}$ (see Sect. 2.2.2).

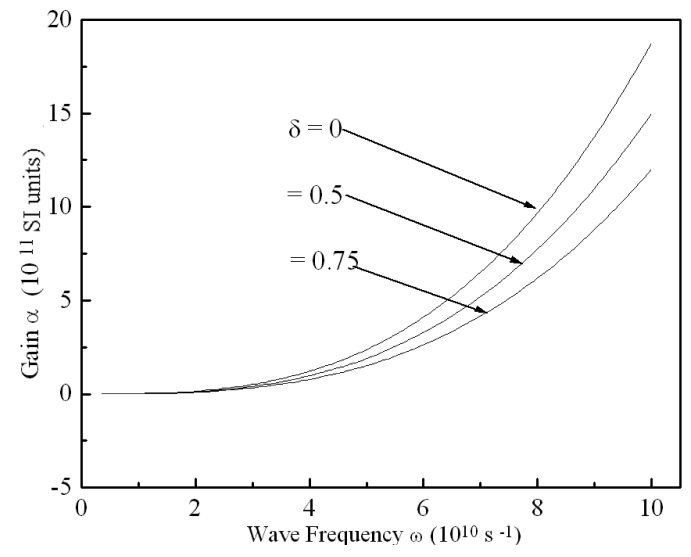

Fig. 4. Variation of gain $\alpha$ with wave frequency $\omega$ using $\delta$ as parameter at $E_{0}=$ $10^{2} \mathrm{~V} \mathrm{~m}^{-1}$ for $\vartheta_{0 e}<\vartheta_{s}<\vartheta_{0 d}$ (see Sect. 2.2.3).

increase in the wave frequency, the gain per radian increases parabolically. But as the uniform medium $(\delta=0)$ changes into the non-uniform one $(\delta \neq 0)$, the value of gain per radian decreases. Hence, in this velocity regime also, the highest value of acoustic gain is possible in a homogeneous medium.

The variation of acoustic gain per radian $\alpha$ with wave frequency $\omega$ using $\delta$ as a parameter is depicted in Fig. 3 when $\vartheta_{0 e}>\vartheta_{s}>\vartheta_{0 d}$. It is clear from the graph that all the curves show identical nature of variations i.e. the gain first increases with wave frequency, attains a maximum value and then starts decreasing with increasing wave frequency. It may be inferred from this figure that the amplification of acoustic wave is possible only when $\omega \leq 1.659 \times 10^{13} \mathrm{~s}^{-1}$. The maximum gain obtained decreases with increase in $\delta$. The frequency at which one obtains maximum gain shifts slightly towards higher frequency point as $\delta$ increases. Similar 
to the first case, here also one gets largest gain only in a homogeneous medium. Hence inhomogeneity due to the density gradient is found to be unfavorable for acoustic wave amplification in ion-implanted piezoelectric semiconductor.

Figure 4 displays the gain per radian $(\alpha)$ versus wave frequency $\omega$ using density gradient $(\delta)$ as parameter when $\vartheta_{0 e}<\vartheta_{s}<\vartheta_{0 d}$. In this velocity regime amplification of acoustic wave is possible only when $\omega>4.217 \times 10^{9} \mathrm{~s}^{-1}$. In the present case even though the drift velocity of electron is smaller than the acoustic phonon speed one obtains gain for the acoustic mode, which is a very fascinating result, obtained in this study. Here in the lowest part of wave frequency, the gain is found to be insensitive while towards higher frequency it increases very sharply with frequency.

It is found that the frequency regime for which one gets amplification is different for different cases studied. The range of gain value is found to be maximum for case in Sect. 2.2.1 when $\vartheta_{0 e}>\vartheta_{s}<\vartheta_{0 d}$ and it is minimum for case in Sect. 2.2.3 when $\vartheta_{0 e}<\vartheta_{s}<\vartheta_{0 d}$. It is true that one obtains minimum gain when electron drift is slower than acoustic speed, but for ordinary sample in this limit acoustic mode is always found to be decaying.

\section{Conclusion}

Hence in this paper we have investigated a novel possibility of acousto-electric wave interaction in non-magnetized inhomogeneous piezoelectric semiconductor. It is concluded from the above study that growth rate may be increased by decreasing the density decay length for the same system parameters. Thus the choice of homogeneous medium is favorable in achieving higher acoustic gain per radian in ion-implanted piezoelectric semiconductor. Since under crossed field configuration complete homogeneity of a plasma system cannot be achieved due to the Lorentz force, one should consider the inhomogeneity of the system while studying the propagation characteristics and instability of electromagnetic wave. Thus for the experimental verification of our theoretical idea, we propose to initiate a serious laboratory experimental efforts.

\section{Acknowledgments}

One of the authors (P.K.) is indebted to Ms. Preeti Thakur for many stimulating discussions.

\section{References}

[1] C.W. White, J.D. Budal, J.G. Zhu, S.P. Withrow, Appl. Phys. Lett. 68, 2389 (1996).

[2] R.F. Hagluna Jr., L.Yang, R.H. Magrunder III, J.E. Witting, K. Becker, R.A. Zuhr, Opt. Lett. 18, 373 (1993).

[3] S.M. Sze, Semiconductor Devices: Physics and Technology, Wiley, India 2002.

[4] S. Ghosh, G.R. Sharma, Pragati Khare, M. Salimullah, Physica B 351, 163 (2004). 
[5] S. Ghosh, G.R. Sharma, M. Salimullah, Physica B 355, 37 (2004).

[6] S. Ghosh, Preeti Thakur, Eur. Phys. J. D 31, 85 (2004).

[7] S. Ghosh, Pragati Khare, Eur. Phys. J. D, in press.

[8] M. Kogami, S. Tanaka, J. Phys. Soc. Japan 30, 775 (1970).

[9] A.G. Mishin, P.E. Zilberman, Yu.G. Bachin, Sov. Phys. Semicond. 8, 1075 (1975).

[10] B. Shapiro, H.N. Spector, Phys. Status Solidi A 29, K155 (1975).

[11] S.K. Sharma, S.P. Singh, J. Phys. Soc. Japan 40, 1056 (1976).

[12] T. Tiedje, R.R. Haering, Canad. J. Phys. 54, 1454 (1976).

[13] R.H.C. Newton, D.M. Short, J. Phys A 6, 722 (1973).

[14] S. Guha, S. Ghosh, Phys. Status Solidi B 87, K9 (1978); 89, K171 (1978).

[15] S. Guha, S. Ghosh, Phys. Status Solidi A 49, 363 (1979).

[16] Ramchandra, J.S. Verma, Indian J. Pure Appl. Phys. 12, 235 (1974).

[17] S. Guha, S. Ghosh, Phys. Status Solidi B 92, K95 (1979).

[18] S. Guha, S. Ghosh, N. Apte, Phys. Lett. A 7, 382 (1979).

[19] K.N. Ostrikov, S.V. Vladimirov, M.Y. Yu, G.E. Morfill, Phys. Plasmas 7, 461 (2000).

[20] M.C. Steele, B. Vural, Wave Interactions in Solid State Plasmas, Mc-Graw Hill, New York 1969, p. 134.

[21] D.L. White, J. Appl. Phys. 33, 2547 (1962).

[22] U. de Angelis, Phys. Scr. 45, 465 (1992). 